\title{
EDITORIAL
}

\section{Chronic myeloid leukemia - some topical issues}

Leukemia (2007) 21, 1347-1352; doi:10.1038/sj.leu.2404733;

published online 10 May 2007

There has been much recent progress in our understanding of the biology of chronic myeloid leukemia (CML). The introduction to the clinic of imatinib mesylate (IM) in 1998 marked the start of a new era in its management. A number of clinicians and basic scientists working in different areas related to CML met in Bermuda from 16 to 18 December 2006. Here, we review briefly some of the more topical issues that were addressed in individual presentations or in discussion. These include the role of genomic instability in causing the BCR-ABL fusion and setting the scene for further events, approaches to characterization of the Bcr-Abl signaling network and the possible roles of the LYN gene and the PP2A tumor suppressor gene. The paper also addresses various aspects of treatment with tyrosine kinase inhibitors, including recommended initial dosage, possible cardiotoxicity, defining response and methods for monitoring responses.

One of the unresolved mysteries in CML and presumably in other malignant conditions is the 'cause' or 'causes' of the initiating lesion and the apparent consequent predisposition to acquire further molecular abnormalities. This presumed predisposition to acquisition of an initiating lesion and then further molecular lesions has been termed 'genomic instability'.

Reciprocal chromosomal translocations may arise as a result of unfaithful repair of spontaneous DNA double-strand breaks (DSBs), most probably induced by oxidative stress, radiation, genotoxic chemicals and/or replication stress. When genes encoding tyrosine kinases are targeted by these mechanisms, the result may be generation of chimeric genes encoding fusion tyrosine kinases, such as $B C R-A B L$. The Bcr-Abl oncoprotein displays transforming activity owing to its constitutive kinase activity, which results in deregulated proliferation, apoptosis, differentiation and adhesion.

The Bcr-Abl kinase may also stimulate the generation of reactive oxygen species leading to enhanced oxidative DNA damage and resulting in numerous DSBs in leukemia cells ${ }^{1}$ In addition, $B C R-A B L$-positive leukemia cells accumulate more DNA lesions, including DSBs after genotoxic treatment. ${ }^{2}$

Unfortunately, the Bcr-Abl kinase compromises the fidelity of DSBs repair mechanisms homologous recombination (HR) and non-homologous end-joining (NHEJ). ${ }^{1,3,4}$ Point mutations and large deletions are introduced into the products of HR and NHEJ in leukemia cells, but not normal counterparts. In addition to faulty DSBs repair, Bcr-Abl reduces the fidelity of nucleotide excision repair (NER) of ultraviolet-induced lesions resulting in point mutations. Mechanisms responsible for introducing point mutations during HR and NER in the leukemia cells are unknown, but overexpression of DNA polymerase- $\beta$ may play an important role. Accumulation of additional genetic abnormalities may lead to resistance to inhibitors, such as IM and malignant progression of the disease.

The current state of knowledge is best summarized by saying that the chimeric $B C R-A B L$ gene is a product of unfaithful repair of two DSBs in genes encoding $B C R$ and $A B L$ resulting in the $t(9 ; 22)$ reciprocal chromosomal translocation. ${ }^{5}$ However, the Bcr-Abl kinase itself enhances genomic instability leading to accumulation of secondary genetic errors, which may be responsible for resistance to small molecule drugs, such as IM, and transformation to blast crisis $(\mathrm{BC}){ }^{6}$

The $B C R-A B L$ signalling network is still incompletely defined and it is entirely possible that the action of established Bcr-Abl inhibitors could be enhanced by combining them with other kinase inhibitors. Conversely, the clinical efficacy in CML of the known Bcr-Abl inhibitors could be due in part to inhibition of kinases other than Bcr-Abl.

Bcr-Abl supposedly serves as a docking scaffold for a distinct set of proteins linking the oncoprotein to diverse signal transduction pathways that are decisive for Bcr-Abl-dependent oncogenic transformation. Although a lot is known about individual binary protein-protein interactions of Bcr-Abl, fundamental questions on the cellular mechanism of action of $\mathrm{Bcr}-\mathrm{Abl}$ are still open, including its critical effectors. ${ }^{7,8} \mathrm{~A}$ systematic and comprehensive approach in a defined cellular setting has been performed aiming at identifying the basic set of Bcr-Abl complex components that interact with Bcr-Abl in a 1:1 or 2:1 stoichiometry that may constitute the core of the Bcr-Abl molecular machine and would thus be necessary for Bcr-Abl's action as an oncogenic signaling initiator. Bcr-Abl inhibitors, like dasatinib, target the Bcr-Abl core complex rather than the isolated protein, inhibit Bcr-Abl's constitutive kinase activity and lead to changes in the Bcr-Abl protein complex composition.

Systematic approaches to identification of major protein targets of drugs in the relevant cells/tissues are clearly needed in order to evaluate potential risks or benefits of kinase inhibitors and probably exploit existing compounds for distinct purposes. Chemical proteomics, consisting of the immobilization of drugs, incubation with cell/tissue extracts and subsequent identification of bound proteins by mass spectrometry, is a comprehensive and unbiased method for this purpose. Using this technology, a large number of kinases that are targeted by dasatinib were identified and validated. Importantly, a class of kinases, previously not known to be inhibited by dasatinib, was shown to be a major target of dasatinib (Hantschel et al., submitted for publication).

One of the major differences between the chronic phase (CP) and $\mathrm{BC}$ phases of $\mathrm{CML}$ is their differential responsiveness to antileukemia treatment including tyrosine kinase inhibitors. Whereas most CP patients respond well to IM and responses are usually durable, the majority of $\mathrm{BC}$ patients respond but then relapse fairly rapidly. However, the fact that the leukemia cell mass is at least initially reduced indicates that the BC cells still depend to some extent on Bcr-Abl kinase activity for their survival. Thus, quantitative rather than qualitative differences in $\mathrm{Bcr}-\mathrm{Abl}$ oncogenic signaling may be responsible for the different responses to IM in the different phases of CML.

The expression of $B C R-A B L$ mRNA and protein is elevated in $\mathrm{CD} 34+$ progenitor cells from BC as compared with CP CML. ${ }^{9}$ In order to assess whether quantitative differences in Bcr-Abl expression could underlie the diverse response of CML CP and $\mathrm{BC}$ cells to IM, Melo et al. studied this phenomenon in $B C R$ $A B L$-transduced $32 \mathrm{D}$ cell clones expressing variable amounts of 
the oncoprotein comparable with those observed in primary CML cells. The main conclusion was that the rate at which an IM-resistant subclone emerged was dependent on the basal level of Bcr-Abl expression by the cell: the higher this level, the faster IM resistance developed. These results suggested that this rate might be unaffected by the mechanism of resistance, and that the first response of a leukemic clone to exposure to IM was upregulation of Bcr-Abl expression. Furthermore, the degree of such upregulation seemed to be inversely proportional to the basal level of Bcr-Abl expression. The presence of high oncoprotein levels in the selected subclone might increase the overall rate of DNA mis-repair ${ }^{6}$ and thus allow rare cells carrying 'resistance-endowing' mutations to survive and proliferate in the presence of IM.

$L Y N$ is a member of the Src-family of tyrosine kinases (SFK) and plays an important role in signal transduction, development and tumorigenicity. In normal development, $L Y N$ functions to attenuate B-cell signaling by recruiting tyrosine phosphatases to the immune synapse. Loss-of-Lyn function leads to multiple changes in hematopoiesis, most commonly manifest in lymphoproliferative disorders. ${ }^{10}$ However, Lyn and other closely related SFKs (Hck, Fyn) play a distinct role in specific leukemias where they appear to function in leukemogenesis and transformation. In CML cells, Lyn and Hck are activated by Bcr-Abl ${ }^{11}$ and Bcr-Abl-induced transformation requires Lyn or a related kinase for leukemogenesis in the lymphoid compartment. ${ }^{12}$ There is emerging evidence for functional cross-talk between the SFKs and the Bcr-Abl kinase; ${ }^{13}$ there may be a role for SFKs in regulation of $\mathrm{CML}$ survival ${ }^{14}$ and increasing the sensitivity of CML cells to tyrosine kinase inhibitors. ${ }^{15}$ Therefore, targeted inhibition of Lyn and other members of this kinase family may provide effective therapy for many leukemias, including CML.

$\mathrm{IM}$ is frontline therapy for $B C R-A B L$-expressing leukemias but resistance is reported in some early phase patients and is common in advanced disease. Resistance is generally associated with mutations in the $B C R-A B L$ kinase domain that affect drug affinity but increasingly patients are reported who fail IM therapy while retaining unmutated $B C R-A B L$ expression. Previous studies suggested a role for Lyn in IM resistance. K562 cells selected for IM resistance (K562R) overexpress Lyn kinase and its targeted silencing overcomes IM resistance and engages apoptosis. Overexpression of Lyn in K562 cells reduces IM sensitivity (threefold) and patients who fail IM therapy in the absence of BCR-ABL mutations express a highly activated Lyn kinase. While Lyn is activated by Bcr-Abl in IM responsive CML patients, it is Bcr-Abl independent in IM resistant patients and is not suppressed by IM. Silencing Lyn expression in patient specimens induces changes in cell survival that are proportional to the level of Lyn protein reduction. To understand the role of Lyn kinase in IM resistance and apoptosis, Donato et al. examined proteins associated with this kinase in IM resistant cell lines, in leukemic cells overexpressing Lyn and in specimens derived from IM resistant patients (Wu et al., Blood 2006; 108: 604a, abstract). Lyn overexpression blocked complete suppression of Bcr-Abl tyrosine phosphorylation (Y177) by IM and affected Bcr-Abl signaling adaptors. Although Bcr-Abl forms a stable complex with the leukemogenic-critical adaptor protein Gab2 in imatinib sensitive cells, Lyn overexpression resulted in the formation of Lyn:Gab2:Bcr-Abl complexes in resistant cells. $\mathrm{Bcr}-\mathrm{Abl}$ kinase inhibition failed to induce tyrosine phosphorylation of Gab2 in these cells, while Lyn silencing (small interfering RNA) or kinase inhibition (with dasatinib) completely suppressed Gab2 tyrosine phosphorylation and correlated with the induction of apoptosis. Lyn silencing in K562R cells also led to a reciprocal increase in the tyrosine phosphorylation and associa- tion with a protein of $\sim 120 \mathrm{kDa}$, identified as the E3 ligase, Cbl. Lyn overexpression in K562 cells reduced both IM sensitivity and $\mathrm{Cbl}$ protein levels. Kinase inhibitor and co-transfection studies demonstrated that tyrosine phosphorylation of $\mathrm{Cbl}$ at a critical signaling site (Y774) is primarily controlled by Bcr-Abl and deletion or mutation of the Cbl RING domain altered its $\mathrm{Bcr}-\mathrm{Abl}$-directed phosphorylation. These results suggest that $\mathrm{Cbl}$ complexes are regulated at both the protein and phosphorylation levels by Lyn and Bcr-Abl kinase activities, respectively. Overexpression and/or activation of Lyn may disrupt the balance and regulation of critical regulators of leukemogenic signaling (Gab2) or protein trafficking and stability (Cbl), resulting in increased cell survival and reduced responsiveness to Bcr-Abl kinase inhibition.

CML-BC and Ph-positive acute lymphoblastic leukemia (ALL) are both BCR-ABL-driven leukemias (see above) in which current therapy with Abl kinase inhibitors fails to induce longterm responses; the majority of patients are either primarily refractory or relapse after a few months of treatment. One possible mechanism of resistance may be functional loss of the PP2A tumor suppressor gene. This occurs during CML disease progression and requires increased $B C R-A B L$ expression that, in turn, enhances expression of the potent PP2A inhibitor SET. ${ }^{16}$ Moreover, molecular or forskolin-mediated restoration of PP2A activity impairs in vitro and in vivo $\mathrm{BCR}-\mathrm{ABL}$ leukemogenesis by inducing the SHP-1-dependent inactivation and proteasome degradation of BCR-ABL. ${ }^{16}$

For these reasons Perrotti and co-workers assessed the therapeutic potential of the PP2A activator FTY720 in CML-BC and Ph-positive patient cells and in in vitro and in vivo models of these $B C R-A B L$-positive leukemias. FTY720, a synthetic myriocin analogue structurally similar to sphingosine, is a water soluble non-toxic drug with high oral bioavailability that reversibly arrests lymphocyte trafficking (mainly $\mathrm{CD}^{+} \mathrm{T}$ cells). ${ }^{17}$

FTY720 treatment inhibits proliferation and impairs viability of p210 and p190 BCR-ABL ${ }^{+}$cells with an $\mathrm{EC}_{50}(48 \mathrm{~h})=80 \mathrm{nM}$ (Santhanam et al., Blood 2006; 108; 89a, abstract). Moreover, FTY720 treatment does not alter viability of non-transformed hematopoietic primary cell and cell lines but markedly impairs that of $B C R-A B L$-transformed cells (Santhanam et al., Blood 2006; 108; 89a, abstract). In fact, FTY720 induces caspasedependent apoptosis and markedly impairs the clonogenic potential of IM/dasatinib-sensitive and -resistant (T315I) p210 and p190 Bcr-Abl-expressing myeloid and lymphoid progenitor cell lines and of primary bone marrow $\mathrm{CML}-\mathrm{BC}^{\mathrm{CD} 34+}, \mathrm{CML}-$ $\mathrm{BC}(\mathrm{T} 315 \mathrm{I})^{\mathrm{CD} 34+}, \mathrm{CML}-\mathrm{CP}^{\mathrm{CD} 34+}, \mathrm{CML}^{\mathrm{CP}}(\mathrm{T} 315 \mathrm{I})^{\mathrm{CD} 34+}$ and $\mathrm{Ph}+\mathrm{ALL}{ }^{\mathrm{CD} 34+} / \mathrm{CD} 19+$ patients cells. ${ }^{18}$ Interestingly, the cytokine (IL-3 or IL-7)-dependent growth and differentiation of normal $\mathrm{CD} 4^{+}$myeloid and $\mathrm{CD} 34^{+} / \mathrm{CD} 19^{+}$lymphoid progenitors are not affected by FTY720 treatment (Santhanam et al., Blood 2006; 108: 89a, abstract).

Furthermore, pharmacologic non-toxic doses, ${ }^{19,20}$ of FTY720 markedly suppress leukemogenesis in severe combined immunodeficient mice transplanted with myeloid and lymphoid progenitor cells transformed with $\mathrm{p} 210^{\mathrm{BCR} / \mathrm{ABL}}$ and $\mathrm{p} 190^{\mathrm{BCR} / \mathrm{ABL}}$, respectively., ${ }^{20}$ In fact, at 25 weeks of treatment, the median survival has not yet been reached in FTY720-treated $(10 \mathrm{mg} / \mathrm{kg} /$ day) $\mathrm{BCR} / \mathrm{ABL}^{+}$cell-injected mice. Conversely, all of untreated 32D-p210 ${ }^{\mathrm{BCR} / \mathrm{ABL}} 32 \mathrm{D}-\mathrm{p} 210^{\mathrm{BCR} / \mathrm{ABL}}(\mathrm{T} 315 \mathrm{I})$ and BaF3-p190 ${ }^{\mathrm{BCR} /}$ ${ }^{A B L}$ leukemic mice died of an overt acute leukemia-like process with a median survival of $4.3,4.8$, and 4.1 weeks, respectively $(P<0.001)$. After 25 weeks of FTY720 treatment, 80 and $90 \%$ of p210 and p190 mice, respectively, were alive and in molecular remission. Moreover, long-term (189 days) FTY720 daily 
administration did not induce any adverse effect, and achieved sustained absence of $\mathrm{BCR}-\mathrm{ABL}^{+}$cells in $50 \%$ of mice transplanted with myeloid progenitors expressing the $\mathrm{IM} /$ dasatinib-resistant T315l p210 ${ }^{\mathrm{BCR}-\mathrm{ABL}}$ mutant (Santhanam et al., Blood 2006; 108; 89a, abstract).

Altogether, these findings not only reinforce the importance of the PP2A tumor suppressor in the biology of Ph-positive leukemias ${ }^{18}$ but, because FTY720 has been shown to be safe in Phase I-III clinical trials for multiple sclerosis and solid organ transplant patients, they strongly support the use of this PP2A activator as a novel therapeutic approach for CML-BC and $\mathrm{Ph}+1 \mathrm{ALL}$ and, perhaps, for other cancers characterized by functional loss of PP2A activity.

There is now general agreement that IM as a single agent is the best initial treatment for the great majority of adult patients who present with CML in CP. This view is based largely on the observation that new patients who received IM as initial treatment in the IRIS study had a best observed rate of complete hematological response (CCyR) of $97 \%$ and Kaplan-Meier estimate of cumulative best rate of complete cytogenetic response of $87 \% .^{21}$ It should be noted parenthetically that the actual proportion of patients in CCYR and still taking IM 5 years after starting the drug is somewhat lower than the $87 \%$ figure for 'cumulative best CCyR'. The degree to which the quantity of residual disease was reduced as assessed by marrow cytogenetics and molecular criteria was inversely related to the probability of survival without progression to advanced phase - in other words, patients who achieved a CCyR at 12 months had a substantially lower chance of disease progression than those who failed to achieve CCyR and those who achieved a major molecular response at 18 months fared better than those who did not. At 5 years, overall survival for patients treated with IM up-front was significantly better than that of historical controls treated with interferon- $\alpha$ or interferon containing combinations. ${ }^{22,23}$

Because of the prognostic criteria impact identified by Sokal et $\mathrm{al}^{24}$ on the probability of response for patients treated by imatinib, a case can be made for offering treatment by allogeneic stem cell transplant (allo-SCT) to newly diagnosed patients provided, they are young enough and have suitable human leukocyte antigen (HLA)-matched donors. This may be true especially if they are 'low risk' for transplant-related mortality as assessed European Group for Blood and Marrow Transplantation (EBMT) criteria. $^{25}$ This transplant option applies particularly to children or other patients under the age of 20 with HLA identical sibling donors, who have an EBMT score of 0 or 1 and might thus expect a post-transplant survival of $80 \%$ of greater.

The conventional initial dose for adults with $\mathrm{CML}$ in $\mathrm{CP}$ is $400 \mathrm{mg}$ daily but the maximal tolerated dose has never been established. It has been recommended however that the dose for an individual patient should not be reduced below $300 \mathrm{mg}$ for fear of expediting development of resistance. Investigators in Houston have studied a series of patients who started treatment with $800 \mathrm{mg}$ daily and demonstrated much more rapid control of blood counts and more rapid reduction in BCR-ABL transcript numbers ${ }^{26}$ the incidence of molecular negativity was also higher than in historical control patients treated initially with $400 \mathrm{mg}$ daily. No difference in survival between patients who received $400 \mathrm{mg}$ and those who received $800 \mathrm{mg}$ has yet been demonstrated. As a consequence prospective studies comparing 400 and $800 \mathrm{mg}$ daily have been initiated on both sides of the Atlantic. For patients not entered into such studies, it seems reasonable still to regard $400 \mathrm{mg}$ daily as the appropriate starting dose.

A panel of experts convened under the aegis on the European Leukemia-Net, but comprising persons from other countries also has recently proposed recommendations for defining 'failure' and 'suboptimal response' in patients who started treatment with imatinib for CML in CP. ${ }^{27}$ Patients satisfied one or other of these criteria if they failed to reach specific hematological, cytogenetic or molecular landmarks at specified time points. They could also be classified as a 'failure' if, having reached these landmarks, there was subsequent evidence that their disease was progressing, albeit still consistently with CP disease. The panel also categorized some patients under the heading of 'warnings' if their disease appeared more likely to progress than that of other comparable patients. These recommendations constitute a valuable guide for the clinician treating patients outside the context of a defined clinical trial. They may or may not apply to results of treatment with the newer tyrosine kinase inhibitors.

The Leukemia-Net panel recommended that patients should be monitored by cytogenetic studies performed before the onset of treatment followed by cytogenetic studies of marrow performed at 3 month internals until attainment of CCyR. ${ }^{27}$ BCR-ABL transcript numbers should be measured at 3-month intervals for patients in CCYR and this should be continued indefinitely. It is generally agreed that fluorescence in situ hybridization (FISH) for the BCR-ABL fusion gene may be a valuable adjunct to use of bone marrow cytogenetics until such time as the patient achieves CCYR but thereafter monitoring the patient who continues to respond should be based solely on real-time quantitative-polymerase chain reaction (RQ-PCR), which is far more sensitive than both marrow metaphase cytogenetics and FISH.

Resistance to imatinib may be primary or secondary. Primary resistance whereby a patient never achieves hematologic or cytogenetic remission is relatively uncommon but secondary resistance, where a patient responds initially but then loses his/ her response, is well recognized. A substantial proportion of patients with secondary resistance have Ph-positive subclones bearing point mutations that encode specific amino-acid substitutions, some of which impair the efficiency with which imatinib binds to the ATP-binding pocket of the BCR-ABL kinase. Thus, far about 50 different mutations have been identified and the extent to which they impair the efficiency of imatinib binding varies considerably. ${ }^{28}$ Thus, the substitution of isoleucine for threonine at Abl amino acid position 315 is associated with almost complete resistance to imatinib and the second generation $\mathrm{TKI}^{29}$ whereas other mutations, such as $\mathrm{M} 244 \mathrm{~V}$, are associated only with very variable or only minor impairment of imatinib function. ${ }^{30}$ Moreover, whereas the finding that a mutant subclone constitutes the majority of the Ph-positive transcripts in a patient resistant to imatinib is consonant with the notion that the mutant subclone is the direct cause of the resistance, mutant subclones may also be identified at low level where their clinical significance is doubtful. ${ }^{31}$

This means that the finding of a mutant subclone at relatively high level may be valuable in supporting the clinical decision to change therapy. Conversely, a search for kinase domain mutations in a previously untreated patient or in a patient who continues to respond well to imatinib is unnecessary and probably a waste of money.

A recent report described 10 patients treated with IM who developed cardiac failure within 15 months of starting treatment with IM for CML or other conditions. ${ }^{32}$ Most of the patients had received imatinib at $800 \mathrm{mg}$ daily. Eight of the patients had concomitant disorders that might have predisposed to cardiac failure, including coronary artery disease, hypertension and diabetes mellitus. The paper also reported the results of administering IM to experimental mice, which developed 
morphological abnormalities in myocardial cell mitochondria. Treating cell lines with IM induced similar changes in myocardial cell lines transduced with wild-type c-ABL but cells transduced with an ABL containing the T315I mutation were resistant to $\mathrm{IM}$ damage. The authors concluded that $\mathrm{IM}$ at prolonged dosage could cause myocardial damage.

In fact, there is no evidence from other studies that IM causes or contributes to cardiac failure in man. Data collated by the manufacturer, Novartis Pharma in Basel, in a clinical database comprising 2327 patents treated with IM and representing an estimated 5595 years of patient exposure show that cardiac failure has been reported with a low incidence of $0.04 \%$ per annum and this potential risk is now included with the literature accompanying distribution of the drug. ${ }^{33}$ A recent study from the Houston group in which 1276 patients who received IM with a median follow-up time of 5 years showed 22 patients with symptoms attributable to cardiac failure, an incidence of $1.8 \%$; the median age of the 22 patients was 70 and 18 had other conditions that might have predisposed to cardiac failure. ${ }^{34}$ The authors conclude that IM can be implicated as cause of cardiac failure only rarely. Until such time as further information convincingly incriminating IM is presented, it seems unnecessary to monitor individual patients for possible cardiotoxicity unless they already have cardiac problems or a separate well-defined predisposing factor.

Resistance to IM may be primary or secondary. ${ }^{27}$ Primary resistance defines the relatively uncommon situation where a patient never achieves an adequate response to IM at $400 \mathrm{mg}$ daily or even to a higher dose. Secondary or acquired resistance describes the situation where a patient who has initially achieved a CCyR or a major molecular response subsequently loses this response. Secondary resistance is very frequently caused by point mutations in the tyrosine kinase domain of BcrAbl. Strategies to cope with resistance have led to the development of two second generation Bcr-Abl inhibitors, nilotinib and dasatinib, which have a higher affinity for Bcr$\mathrm{Abl}$, as compared with IM, and inhibit the majority of clinically relevant IM-resistant mutant subclones. ${ }^{29,35,36}$ Nilotinib and dasatinib have similar potencies in inhibiting Bcr-Abl, but significantly differ in their target specificity. Dasatinib inhibits a large number of tyrosine kinases, including the Src family kinases and several receptor tyrosine kinases, whereas nilotinib, like IM, inhibits mainly Abl, C-KIT and PDGFR.

The largest experience with these second-generation TK inhibitors has been gained with dasatinib and nilotinib (Table 1). Both these two agents have been used mostly in the context of patients who are either resistant to or intolerant of IM, and have included patients in all stages of the disease, as well as patients with Ph-positive acute lymphoblastic leukemia. Both agents have resulted in hematologic and cytogenetic responses in a large proportion of patients, particularly those treated still in the $\mathrm{CP}^{37}$ (Le Coutre et al., Blood 2006; 108: 53a, abstract). The reported rate of CCYR in this population is $34 \%$ (nilotinib; median follow-up 9 months) and 49\% (dasatinib; median follow-up 15 months). As with IM, responses have improved over time from the date of the first response. Within the constraints of the short follow-up available, responses achieved in patients with CML in CP have been durable, with $80-90 \%$ of patients free from progression at 12 months. Responses are significantly less durable in the more advanced stages of $\mathrm{CML}$, particularly in the blast phase.

Dasatinib has been recently approved by the FDA and approval for nilotinib is expected soon. An interesting recent observation with dasatinib relates to the optimal dose and schedule to be administered. The approved dose of dasatinib
Table 1 Tyrosine kinase inhibitors and other agents targeting Bcr$\mathrm{Abl}$

\section{Original TK inhibitor} Imatinib

New generation of TK inhibitors

Dasatinib, nilotinib, bosutinib (SKI-606), INNO-406,

Agents active against the T315I

MK-0457, SGX70393

Agents with various other modes of action IPI-504, GNF-2

was $70 \mathrm{mg}$ twice daily based on the lack of dose limiting toxicity up to this dose and the short half-life (approximately $5 \mathrm{~h}$ ) of the drug. However, it appears that a once daily administration might be associated with less toxicity, particularly, a reduced rate of pleural effusions and, in the case of $\mathrm{CP}$, also less myelosuppression. This schedule maintains similar efficacy even at doses of $100 \mathrm{mg}$ daily in CP (Hochhaus et al., Blood 2006; 108: 53a, abstract). Further analysis of results of ongoing studies addressing, among other things, whether the duration of response with different doses might be equivalent, might lead to a change in the recommended dose and schedule in the near future. Studies of these agents in patients with previously untreated $\mathrm{CML}$ in $\mathrm{CP}$ have now been initiated.

During the last 2 years, various other new compounds were developed, which might be valuable for treating CML used either alone or in combination with $\mathrm{IM}$ or with second generation Bcr-Abl inhibitors mentioned above. Thus, agents active against the $\mathrm{Bcr}-\mathrm{Abl}$ oncoprotein can be classified as follows:

(a) New agents with TK inhibitor target profile differing from imatinib (but inactive against T315 subclones). The new agent INNO-406 (previously known as NS-187) is a modified version of imatinib with considerable greater activity than the parent drug. Like dasatinib, it appears also to inhibit the Src family kinase Lyn. ${ }^{38,39}$ Bosutinib (SKI-606) is a new inhibitor that resembles dasatinib in activity against $\mathrm{Abl}, \mathrm{Src}$ and $\mathrm{Src}$ family kinases. ${ }^{40}$ Both agents are active against IM resistant mutant subclones other than the T315I. .

(b) Kinase inhibitors that inhibit the Bcr-Abl T315I mutant clone. Examples include MK-0457 (previously VX-680), which inhibits Bcr-Abl T315I and JAK2 and might display additional benefits as a cytostatic agent by inhibiting Aurora kinases ${ }^{41}$ and SGX70393 that very potently inhibits Bcr-Abl T315I and was identified by a fragment-based structural genomics approach (O'Hare et al., 2006; 108: 400a, abstract).

(c) Compounds that target sites on BCr-Abl other than the ATPbinding pocket. One example of a drug in this category is GNF2 that acts as an allosteric inhibitor targeting the myristoyl binding pocket of Bcr-Abl, which has been identified as an important autoinhibitory site in Abl. ${ }^{42}$

(d) Compounds that destabilize oncogenic tyrosine kinases by targeting the HSP90 molecular chaperone machinery. IPI-504, a derivative of the heat-shock protein-90 inhibitors geldanamycin and $17-\mathrm{AAG}$, is currently the best example. ${ }^{38}$

All these approaches are being evaluated for their clinical benefit, but clearly might lead towards a more individualized treatment of $\mathrm{CML}$ when used alone, in combination with IM or with one of the second generation Bcr-Abl inhibitors.

There is currently no general agreement about how best to treat the patient for whom imatinib is no longer effective. For the patient who fails imatinib and then responds to dasatinib, the durability of response at 1 year seems satisfactory but the 
duration follow-up is limited. ${ }^{35}$ Nilotinib, which should be licensed shortly, would also be a reasonable choice for the patient who fails imatinib. ${ }^{36}$ For the patient who would have been a candidate for allo-SCT in the pre-imatinib era, this option should be considered seriously if he/she fails imatinib. A possible compromise for such patients would be to treat the imatinib failure patient with a second generation TKI for specific period, for example 6 months, and to recommend allo-SCT if the response seems to be inadequate at that time point. Clearly, the patient's preference will be a major determinant of clinical management.

As the projected survival for patients with $\mathrm{CML}$ must now exceed 10 years and may be much longer, and as the number of tyrosine kinase inhibitors available for clinical use increases, the criteria for assessing success or failure in prospective clinical studies need to be re-evaluated. End points such as achievement of cytogenetic or molecular remission become crucial. However, there is at present considerable variability in the way in the numbers of $\mathrm{BCR}-\mathrm{ABL}$ transcripts are measured in different laboratories.

Investigators at a recent meeting in Bethesda, USA recommended some degree of standardization in the methodology and manner of expression of the results of the real-time reverse transcriptase-PCR for BCR-ABL transcripts. ${ }^{43}$ It was recommended, for example, that assays should use one of three possible control genes, $\mathrm{ABL}, \mathrm{BCR}$ or GUSB. It was recommended that results be expressed as leukemia copy numbers/ control copy numbers $\times 100 \%$. The less preferred alternative, which may in fact be more 'user friendly', is to express results as log reduction from a standardized baseline for untreated patients defined at $100 \%$ as developed for the IRIS study. ${ }^{43}$ Using this methodology, a patient in complete cytogenetic remission is likely to have achieved at least a 2 log reduction in BCR-ABL transcripts and a 3 log reduction has been equated with a major molecular response. With a reduction of 4.5-5.0 logs BCR-ABL transcripts can no longer be reliably detected.

Investigators at the Bethesda meeting agreed that collaborating laboratories should derive a laboratory-specific conversion factor to relate values obtained in their laboratory to values determined on an international scale (IS), which is anchored to two key levels used in the IRIS study, ${ }^{44}$ namely, a standardized baseline defined as $100 \% \mathrm{BCR}-\mathrm{ABL}^{\mathrm{IS}}$ and major molecular response (3 log reduction relative to the standardized baseline), defined as $0.1 \% \mathrm{BCR}-\mathrm{ABL}^{\mathrm{IS}}$. The converted value from a given laboratory should then be equivalent to an analogous converted value obtained in any other collaborating laboratory. The strength of this approach is that (i) laboratories can continue to use their existing assay conditions (provided they use at least one of the three control genes above and the assay is linear on analysis of the reference samples) and (ii) that they can continue to express results according to local preferences in addition to expressing results on the international scale.

Currently, derivation of local conversion factors is only possible using a series of reference samples prepared by the two international reference centers in Adelaide or Mannheim. Further reference centers may be established but the intention is to produce widely available, internationally accredited 'higher order' reference materials that testing laboratories can use to derive local conversion factors on demand and calibrate their own local reference materials. This project is proceeding with the intention that these higher-level reference materials can be available for distribution in 1-2 years.

In view of the likelihood that the number of papers summarizing phase 2 and 3 trials submitted for publication in coming years will increase, it was thought that some level of standardization should be sought, so that results with a given drug or drug combination could reasonably be compared with other published results. The following recommendations are suggested:

(a) The study end points should be specified.

(b) The definitions of response and failure should if possible follow agreed criteria.

(c) There should be clear distinction between primary and secondary resistance in patients treated ab initio with a TKI.

(d) The aim should be to follow all patients indefinitely including those who abandon the study for any reason.

(e) Conventional Kaplan-Meier curves should be presented for overall survival and survival subclassified as appropriate for the patient population.

(f) The number of patients at risk at the various time points should be specified.

(g) Presentation of cumulative end points should be supported by presentation of the numbers of patients who maintain a given level of response at the various time time points.

\section{Acknowledgements}

We thank Bristol-Myers Squibb, Novartis Pharmaceuticals and Merck Oncology for unrestricted educational grants in support of the Bermuda meeting.

T Mughal $^{1}$, J Cortes ${ }^{2}, \mathrm{NCP} \mathrm{Cross}^{3}, \mathrm{~N}$ Donato $^{4}, \mathrm{O}$ Hantschel $^{5}$, E Jabbour $^{2}$, H Kantarjian $^{2}$, JV Melo ${ }^{6}$, T Skorski $^{7}$, RT Silver $^{8}$ and JM Goldman ${ }^{6}$

${ }^{1}$ Department of Hematology and Stem Cell Transplantation, University of Texas Southwestern Medical School, Dallas,

Texas USA;

${ }^{2}$ Department of Leukemia, University of Texas MD Anderson Cancer Center, Houston, Texas USA; ${ }^{3}$ Wessex Regional Genetics Laboratory, University of Southampton, Salisbury UK;

${ }^{4}$ Department of Internal Medicine, University of Michigan Health Systems, University of Michigan, Ann Arbor, Michigan USA;

${ }^{5}$ Center for Molecular Medicine of the Austrian Academy of Sciences, Vienna, Austria; ${ }^{6}$ Department of Haematology; Imperial College at Hammersmith Hospital, London, UK; ${ }^{7}$ Department of Microbiology and Immunology, Temple University, Philadelphia, Pennsylvania USA and

${ }^{8}$ Weill Cornell Medical College, New York-Presbyterian Hospital, New York City, NY USA E-mail: jgoldman@imperial.ac.uk

\section{References}

1 Nowicki MO, Falinski R, Koptyra M, Slupianek A, Stoklosa T, Gloc $\mathrm{E}$ et al. BCR/ABL oncogenic kinase promotes unfaithful repair of the reactive oxygen species-dependent DNA double-strand breaks. Blood 2004; 104: 3746-3753.

2 Nieborowska-Skorska M, Stoklosa T, Datta M, Czechowska A, Rink L, Slupianek A et al. ATR-Chk1 axis protects BCR/ABL leukemia cells from the lethal effect of DNA double-strand breaks. Cell Cycle 2006; 5: 994-1000.

3 Slupianek A, Schmutte C, Tombline G, Nieborowska-Skorska M, Hoser G, Nowick MO et al. BCR/ABL regulates mammalian RecA homologs, resulting in drug resistance. Mol Cell 2001; 8: 795-806.

4 Gaymes TJ, Mufti GJ, Rassool FV. Myeloid leukemias have increased activity of the nonhomologous end-joining pathway and concomitant DNA misrepair that is dependent on the Ku70/86 heterodimer. Cancer Res 2002; 62: 2791-2797. 
5 Koptyra M, Falinski R, Nowicki MO, Stoklosa T, Majsterek I, Nieborowska-Skorska $\mathrm{M}$ et al. BCR/ABL kinase induces selfmutagenesis via reactive oxygen species to encode imatinib resistance. Blood 2006; 108: 319-327.

6 Penserga ET, Skorski T. Fusion tyrosine kinases: a result and cause of genomic instability. Oncogene 2006; 26: 11-20.

7 Hantschel O, Superti-Furga G. Regulation of the c-Abl and Bcr-Abl tyrosine kinases. Nat Rev Mol Cell Biol 2004; 5: 33-44.

8 Ren R. Mechanisms of BCR-ABL in the pathogenesis of chronic myelogenous leukaemia. Nat Rev Cancer 2005; 5: 172-183.

9 Barnes DJ, Palaiologou D, Panousopoulou E, Schultheis B, Yong A, Goldman JM et al. Bcr-Abl expression levels determine the rate of development of resistance to imatinib mesylate in chronic myeloid leukemia. Cancer Res 2005; 65: 8912-8919.

10 Xu Y, Harder KW, Huntington ND, Hibbs ML, Tarlington DM. Lyn tyrosine kinase: accentuating the positive and the negative. Immunity 2005; 22: 9-18.

11 Danhauser-Riedl S, Warmuth M, Druker BJ, Emmerich B, Hallek M. Activation of Src kinases p53/56lyn and p59hck by p210bcr/abl in myeloid cells. Cancer Res 1996; 56: 3589-3596.

$12 \mathrm{Hu} \mathrm{Y,} \mathrm{Liu} \mathrm{Y,} \mathrm{Pelletier} \mathrm{S,} \mathrm{Buchdunger} \mathrm{E,} \mathrm{Warmuth} \mathrm{M,} \mathrm{Fabbro} \mathrm{D}$ et al. Requirement of Src kinases Lyn, Hck and Fgr for BCR-ABL1induced B-lymphoblastic leukemia but not chronic myeloid leukemia. Nat Genet 2004; 136: 453-461.

13 Meyn MA, Wilson MB, Abdi FA, Fahey N, Schiavone AP, Wu J et al. Src family kinases phosphorylate the Bcr-Abl SH3-SH2 region and modulate $\mathrm{Bcr}-\mathrm{Abl}$ transforming activity. J Biol Chem 2006; 281: 30907-30916.

14 Ptasznik A, Nakata Y, Kalota A, Emerson SG, Gewirtz AM. Short interfering RNA (siRNA) targeting the Lyn kinase induces apoptosis in primary, and drug-resistant, BCR-ABL1(+) leukemia cells. Nat Med 2004; 10: 1187-1189.

15 Donato NJ, Wu JY, Stapley J, Gallick G, Lin H, Arlinghaus R et al. $\mathrm{BCR}-\mathrm{ABL}$ independence and LYN kinase overexpression in chronic myelogenous leukemia cells selected for resistance to STI571. Blood 2003; 101: 690-698.

16 Neviani P, Santhanam R, Trotta R, Notari M, Blazer BW, Liu S et al. The tumor suppressor PP2A is functionally inactivated in blast crisis CML through the inhibitory activity of the BCR/ABLregulated SET protein. Cancer Cell 2005; 8: 355-368.

17 Kahan BD. FTY720: from bench to bedside. Transplant Proc 2004; 36: 531S-543S

18 Perrotti D, Neviani P. ReSETting PP2A tumour suppressor activity in blast crisis and imatinib-resistant chronic myelogenous leukaemia. Br J Cancer 2006; 95: 775-781.

19 Brinkmann V. FTY720: mechanism of action and potential benefit in organ transplantation. Yonsei Med J 2004; 45: 991-997.

20 Yasui H, Hideshima T, Raje N, Roccaro AM, Shiraishi N, Kumar S et al. FTY720 induces apoptosis in multiple myeloma cells and overcomes drug resistance. Cancer Res 2005; 65: 7478-7484.

21 Druker BJ, Guilhot F, O'Brien SG, Gathamnn I, Kantarjian H, Gattermann $\mathrm{N}$ et al. Five-year follow-up of imatinib therapy for newly diagnosed chronic myeloid leukemia in chronic-phase shows sustained responses and high overall survival. N Engl J Med 2006; 355: 2408-2417.

22 Roy L, Guilhot J, Krahnke T, Guerci-Bresler A, Druker BJ, Larson RA et al. Survival advantage with imatinib compared to the combination interferon-a plus cytarabine in chronic phase CML: historical comparison between two phase III trials. Blood 2006; 108: $1478-1484$.

23 Kantarjian H, Talpaz M, O'Brien S, jones D, Giles F, GarciaManero D et al. Survival benefit with imatinib mesylate versusinterferon alfa-based regimens in newly diagnosed chronic phase chronic myelogenous leukemia. Blood 2006; 108: 1835-1840.

24 Sokal JE, Cox EB, Baccarani M, Tura S, Gomez GA, Robertson JE et al. Prognostic discrimination in 'good risk' chronic granulocytic leukemia. Blood 1984; 63: 789-799.

25 Gratwohl A, Hermans J, Goldman JM, Arcese W, Carreras E, Devergie A et al. Risk assessment for patients with chronic myeloid leukaemia before allogeneic blood or marrow transplantation. Chronic Leukemia Working Party of the European Group for Blood and Marrow Transplantation. Lancet 1998; 352: 1087-1092.
26 Kantarjian H, Talpaz M, O'Brien S, Garcia M, Verstovsek S, Giles F et al. High dose imatinib mesylate therapy in newly diagnosed Philadelphia chromosome-positive chronic phase chronic myeloid leukemia. Blood 2004; 103: 2873-2878.

27 Baccarani M, Saglio G, Goldman JM, Hochhaus A, Simonsson B, Appelbaum $\mathrm{F}$ et al. Evolving concepts in the management of chronic myeloid leukemia. Recommendations from an expert panel on behalf of the European Leukemia-net. Blood 2006; 108: 1809-1820.

28 Corbin AS, La Rosee P, Stoffregen EP, Druker BJ, Deininger MW. Several Bcr-Abl kinase domain mutations associated with imatinib mesylate resistance remain sensitive to imatinib. Blood 2003; 101 4611-14614.

29 Shah NS, Tran C, Lee FY, Chen P, Norris D, Sawyers CL. Overriding imatinib resistance with a novel Abl kinase inhibitor. Science 2004; 305: 399-401.

30 Anand M, Khoramshad JS, Marin D, Apperley JF, Goldman JM, Kaeda JS. Varying response to escalating the dose of imatinib in CML patients who acquire a BCR-ABLM244V mutation. Blood 2006; 108: 2881-2882.

31 Khorashad J, Anand M, Marin D, Saunders S, Al-Jabary T, Iqbal A et al. The presence of a bcr-abl mutant allele in $\mathrm{cml}$ does not always explain clinical resistance to imatinib. Leukemia 2006; 20: 658-663.

32 Kerkela R, Grazette L, Yacobi R, Iliescu C, Patten R, Beahm C et al. Cardiotoxicity of the cancer therapeutic agent imatinib mesylate. Nat Med 2006; 12: 908-916.

33 Hatfield A, Owen S, Pilot PR. In reply to 'Cardiotoxicity of the cancer therapeutic agent imatinib mesylate'. Nat Med 2007; 13: 13.

34 Atallah E, Kantarjian H, Cortes J. In reply to 'Cardiotoxicity of the cancer therapeutic agent imatinib mesylate'. Nat Med 2007; 13: 14.

35 Talpaz M, Shah NP, Kantarjian H, Donato N, Nicoll J, Paquette R et al. Dasatinib in imatinib-resistant Philadelphia chromosomepositive leukemias. N Engl J Med 2006; 354: 2531-2541.

36 Kantarjian H, Giles F, Wunderle L, Bhalla K, O'Brien S, Wassmann $\mathrm{B}$ et al. Nilotinib in imatinib-resistant $\mathrm{CML}$ and Philadelphia-chromosome positive ALL. N Engl J Med 2006; 354: 2542-2551.

37 Hochhaus A, Kantarjian H, Baccarani M, Lipton JH, Apperley JF, Druker BJ et al. Dasatinib induces notable hematologic and cytogenetic responses in chronic phase chronic myeloid leukemia after failure of imatinib therapy. Blood 2007; 109: 2303-2307.

38 Guo F, Rocha K, Bali P, Pranpat M, Fiscus W, Boyapalle S et al. Abrogation of heat shock protein 70 induction as a strategy to increase antileukemia activity of heat shock protein 90 inhibitor 17-allylamino-demethoxy geldanamycin. Cancer Res 2005; 65: 10536-10544.

39 Yokota A, Kimura S, Masuda S, Ashihara H, Ruroda J, Sato K et al. INNO-406, a novel Bcr-Abl/Lyn dual tyrosine kinase inhibitor, suppresses the growth of $\mathrm{Ph}+$ leukemia cells in the central nervous system, and cyclosporine A augments its in vivo action. Blood 2007; 109: 306-314.

40 Puttini M, Coluccia AM, Boschelli F, Cleris L, Marchesi E, DonellaDeana A et al. In vitro and in vivo activity of SKI-606, a novel Src$\mathrm{Abl}$ inhibitor, against imatinib-resistant $\mathrm{Bcr}-\mathrm{Abl}+$ neoplastic cells. Cancer Res 2006; 66: 11314-11322.

41 Giles FJ, Cortes J, Jones D, Bergstrom D, Kantarjian H, Freedman SJ. MK-0457, a novel kinase inhibitor, is active in patients with chronic myeloid leukemia or acute lymphocytic leukemia with T315I BCR-ABL mutation. Blood 2007; 109: 500-502.

42 Adrian FJ, Ding Q, Sim T, Velentza A, Sloan C, Liu Y et al. Allosteric inhibittors of Bcr-abl-dependent cell proliferation. Nat Chem Biol 2006; 2: 95-102.

43 Hughes T, Deininger M, Hochhaus A, Branford S, Radich J, Kaedfa J et al. Monitoring CML patients responding to treatment with tyrosine kinase inhibitors - recommendations for 'harmonizing' current methodology for detecting BCR-ABL transcripts and kinase domain mutations and for expressing results. Blood 2006; 108: $28-37$.

44 Hughes TP, Kaeda J, Branford S, Rudzki Z, Hochhaus A, Hensley $\mathrm{ML}$ et al. Frequency of major molecular responses to imatinib or interferon alfa plus cytarabine in newly diagnosed chronic myeloid leukemia. N Engl J Med 2003; 349: 1421-1430. 